\title{
ON THE EQUILIBRIUM OF A FLUID MASS AT REST*
}

BY

\section{JAMES W. ALEXANDER}

1. The following question was brought up a considerable time ago by both Liapounoff $\dagger$ and Poincaré, $\ddagger$ but has apparently not been answered up to the present:

"Consider a homogeneous incompressible fluid whose particles attract one another according to Newton's law and which is acted on by no external forces. Then, are there any positions of equilibrium for the fluid besides the sphere?"

It will be shown that there are no such positions, whether of stable or unstable equilibrium.

2. A necessary condition for equilibrium will be obtained by examining an approximating figure made up of elementary parallelepipeds, or parallel rods. The rods will be treated as rigid and free to move in the direction of their lengths only, so that perpendicular distances between them remain unchanged. They will be so chosen that whenever two collinear rods are moved into contact with one another their ends will fit together exactly and the rods will become merged into one.

If the approximating system consists of two rods only, it can be seen by inspection that its potential energy diminishes continuously as the centers of the rods approach one another. Equilibrium can, therefore, only occur if the rods are touching end to end or if they are symmetrical about a perpendicular line through their centers.

If there are more than two rods, the potential energy of the approximating system is equal to the sum of the potential energies of all sub-systems consisting of two rods only. Suppose the rods are set in motion in such a way that the center of each rod approaches a fixed perpendicular plane, $\pi$, with a velocity equal to its instantaneous absolute distance from $\pi$. Then, as the system moves, the distance between the centers of two rods never increases, while, on the contrary, it decreases whenever the centers are at unequal distances from $\pi$.

\footnotetext{
* Presented to the Society, February 28, 1920.

† Liapounoff, Sur le corps du potentiel maximum, Communications de la Société Mathématique de Kharkow (1887), pp. 63-73.

$\ddagger$ Poincaré, Sur un théorème de M. Liapounoff relatif a l'equilibre d'une masse fluide, $\mathrm{C}$ o $\mathrm{m}$ p t es Rendus, vol. 104 (1887), p. 622.
} 
The rate-loss of potential energy, $-d W / d t$, changes abruptly whenever two collinear rods merge into one, since the rods undergo a sudden change of velocity at that instant. The value of $-d W / d t$ stays greater than zero, however, so long as two rods are collinear without touching and so long as the system is not symmetrical about a plane through its center of mass and parallel to $\pi$. Under either of these conditions, therefore, the approximating system can not be in equilibrium.

3. The problem for the fluid mass itself can now be solved by an obvious passage to the limit if we impose upon the boundary the restrictions that are usually assumed in a discussion of this kind. As we wish to handle the perfectly general case, however, we merely observe at this point that the limiting process is obvious provided the fluid is convex. Therefore, since the orientation of the rods of the approximating systems does not affect the argument in any way, we have the

THEOREM. A convex figure of equilibrium is symmetrical about every plane through its center of mass and is therefore bounded by a sphere.

It remains to be shown that a figure which is not convex cannot be one of equilibrium.

4. Although a perfectly general boundary will be permitted, spurious "boundaries" either wholly within or wholly without the fluid are to be excluded. We therefore assume: that every interior point is within a sphere which encloses interior points only; that every exterior point is within a sphere which encloses exterior points only; that within every sphere about a boundary point there are both interior and exterior points. We shall also assume that there is an upper bound, $L$, to the distance between two interior points.

Under the above assumptions, we must be prepared to meet the case where the boundary of the fluid is a set of points of measure greater than zero, so that the volume depends on whether we decide to count in or leave out the boundary points. ${ }^{*}$ Let us agree to define the volume, $T$, as the measure of the interior points only. The potential energy will then be the Lebesgue integral

$$
\dot{W}=-\frac{k}{2} \iint \frac{d \tau d \tau^{\prime}}{R\left(\tau, \tau^{\prime}\right)}
$$

extended over the interior points, where $k$ is the gravitational constant and $R\left(\tau, \tau^{\prime}\right)$, the ultimate distance between the elements $d \tau$ and $d \tau^{\prime}$. If space be cut up into cubes by means of three systems of parallel planes, the sum of the volumes of the cubes that are wholly within the fluid approaches the limit $T$ as the distance between parallel planes approaches zero. Moreover,

* For the analogous case in two dimensions, cf. Osgood, $A$ Jordan curve of positive area, these Transactions, vol. 4 (1903), pp. 107-112. 
the potential energy of the system composed of interior cubes approaches the limit $W$.

5. Since the fluid is not convex, a segment $P Q P^{\prime}$ can always be found such that $P$ and $P^{\prime}$ are both within the fluid while $Q$ is without it or on the boundary. Moreover, we can always arrange to make $Q$ an outside point by displacing the segment $P Q P^{\prime}$ a bit, if necessary.

Let us approximate the fluid figure, which we shall call $F$, by a sequence of figures, $F_{1}, F_{2}, F_{3}, \cdots$ made up of rods parallel to the segment $P Q P^{\prime}$, such that $P$ and $P^{\prime}$ are interior points of $F_{1}$ and that every figure $F_{i}$ is contained within all subsequent figures of the series and in $F$. Then, to be sure, a plane $\pi$ perpendicular to $P Q P^{\prime}$ can be chosen with reference to which the figures $F_{i}$ can be set in motion in the manner described in $\S 2$. Furthermore a perfectly unambiguous limiting motion can be determined by allowing $i$ to increase indefinitely. Unfortunately, the limiting motion so changes the internal structure of the fluid by transforming interior points into boundary points and vice versa, that we cannot be sure among other things that the volume remains constant. We therefore proceed in a more roundabout way.

About the points $Q, P$, and $P^{\prime}$ we draw spheres of radii $l, l / 3$, and $l / 3$ respectively, where $l$ is so small that the sphere about $Q$ is exterior to the fluid $F$, while the spheres about $P$ and $P^{\prime}$ are interior to the first approximating figure $F_{1}$. We can then obtain a lower bound for the rate-loss of potential energy of the figure $F_{i}$ when set in motion in the way described above, such that this lower bound depends on $l$ and $L(\$ 4)$ alone and not on $i$.

Let us choose the plane $\pi$ perpendicular to $P Q P^{\prime}$ and through the center of mass of $F$. Then consider what happens as the motion begins. The spheres about $P$ and $P^{\prime}$ have between them a gap of exterior points interior to the sphere about $Q$ and of width at least $l$. Consequently, they approach one another with a velocity greater than $l$. Moreover their mutual attraction is at least $k\left(T^{2} / L^{2}\right)$, where $T$ is the volume of each.

Therefore, if the potential energy of $F_{i}$ be $W_{i}$, we have at the start of a motion

$$
-\frac{d W_{i}}{d t}>\phi, \quad \text { where } \quad \phi=k \frac{T^{2}}{L^{2}} l
$$

while during an interval of time $\bar{t}$ depending on $l$ and $L$ but not on $i$, we have

$$
-\frac{d W_{i}}{d t}>\phi / 2, \quad \bar{t}>t>0 \text {. }
$$

Suppose now, we expand the figures $F_{i}$ to the volume of $F$ by similarity transformations which preserve directions and leave fixed the center of mass of $F$. We then obtain a second sequence of approximating figures $F_{i}^{\prime}$ of the 
same volume as $F$. Moreover, when the figures $F_{i}^{\prime}$ are set in notion in the same way as the figures $F_{i}$, we have from (1)

$$
-\frac{d W_{i}^{\prime}}{d t}>\phi / 2, \quad \bar{t}>t>0
$$

\section{a fortiori.}

Let us denote by $\epsilon_{i}^{\prime}$ the maximum displacement undergone by any point of the figure $F_{i}^{\prime}$ after a motion lasting a time $t$. Then we have

and therefore,

$$
L d t \geqq d \epsilon_{i}^{\prime},
$$

$$
-\frac{d W_{i}^{\prime}}{d \epsilon_{i}}>\frac{\phi}{2 L}, \quad \bar{t}>t>0 .
$$

Furthermore, if $\Delta W_{i}^{\prime}$ be the total change in potential energy of the figure $F_{i}^{\prime}$ after motion lasting a time $t$ and if $\Delta s_{i}^{\prime}$ be the maximum distance from a point on the interior or boundary of the displaced figure to the nearest point on the interior or boundary of the figure in its original position, we have

Therefore

$$
\Delta W_{i}^{\prime}=\int_{0}^{t} d W, \quad \text { and } \quad \Delta s_{i}^{\prime} \leqq \int_{0}^{t} d \epsilon_{i}^{\prime} .
$$

$$
-\frac{\Delta W_{i}^{\prime}}{\Delta s_{i}^{\prime}}>\frac{\phi}{2 L}
$$

Now, let $\Delta \bar{W}_{i}$ be the difference between the potential energy of the displaced figure $F_{i}^{\prime}$ at a time $t$ and the potential energy of the fluid $F$, and let $\Delta \bar{s}_{i}$ be the maximum distance from a point on the interior or boundary of the displaced $F_{i}$ and the nearest point on the interior or boundary of $F$. Then, if we hold $t$ fixed, we have

$$
\lim _{i=\infty}\left[\frac{\Delta W_{i}^{\prime}}{\Delta s_{i}^{\prime}}-\frac{\Delta \bar{W}_{i}}{\Delta \bar{s}_{i}}\right]=0,
$$

since the undisplaced figure $F_{i}$ tends towards the figure $F$ in the limit. Therefore by (3) and (4), we can find, corresponding to any $t$, a value of $i$ such that

$$
-\frac{\Delta \bar{W}_{i}}{\Delta \bar{s}_{i}}>\frac{\phi}{2 L} \text {. }
$$

Finally, let $t$ run through a decreasing sequence of values with zero as limit. Then, corresponding to each value of $t$, we can find a displaced figure $F_{i}$ such that the relation (5) holds. These displaced figures evidently approach $F$ as limit, since $i$ increases and the time of displacement tecreases towards zero. Therefore, in view of (5), the figure $F$ is not in equilibrium. Hence:

The sphere is the only figure of equilibrium. 
6. Before leaving this matter, let us say one word about the more general problem, where the fli:d mass is rotating about an axis through its centey of gravity. The methods used in this paper show us at once that the fluid cannot be in equilibrium unless symmetrical about a plane perpendicular to the axis of rotation and unless a line parallel to this axis cuts the fluid in at most one segment.

New York, January, 1919. 